\title{
Práctica por computadora y aprendizaje del idioma inglés
}

\author{
Vilma Lisseth Majano García
}

Maestra en Asesoría Educativa

Docente investigadora, Facultad Multidisciplinaria de Ilobasco, Cabañas

Universidad Católica de El Salvador, El Salvador

Email: vilma.majano@catolica.edu.sv

Fecha de recepción: 21-12-2019

ORCID: https://orcid.org/0000-0002-1799-3833

Fecha de aceptación: 15-02-2020

\section{Resumen}

El objetivo principal del estudio fue desarrollar prácticas de lectura y audio a través de la computadora, para que los estudiantes de Inglés Intermedio I de la Licenciatura en Ciencias de la Educación con especialidad en Idioma Inglés de la Universidad Católica de El Salvador, Facultad Multidisciplinaria de Ilobasco, Cabañas, desarrollaran sus habilidades en comprensión de vocabulario, lectura y audio, en aras de un mejor rendimiento en el examen estandarizado TOEIC (Test of English for International Communication).

La población de estudiantes de Inglés Intermedio I fue dividida en dos grupos: un grupo fue de control y el otro de intervención. Para este último grupo, se presentaron semanalmente un texto y un audio. Con base en ellos se desarrollaron exámenes de doce preguntas (de comprensión lectora y auditiva) con diferentes niveles de dificultad. Como resultado, el $60 \%$ de los estudiantes expuestos a las prácticas alcanzaron un puntaje de 280 a 500 puntos en el examen estandarizado TOEIC.

Palabras clave: CALL, práctica, TOEIC, hábito, frecuencia, comprensión.

\begin{abstract}
The main objective of the study was to develop reading and audio practices through the computer so that students of Intermediate English I of the Bachelor of Arts with specialization in English Language from Universidad Católica de El Salvador, Multidisciplinary Faculty of Ilobasco, Cabañas, will develop their skills in vocabulary comprehension, reading and audio, in order to improve their performance on the standardized exam, TOEIC (Test of English for International Communication)

The population of Intermediate English I students was divided into two groups: one group was control and the other was intervention. For this last group, a text and an audio were presented weekly. Based on them, tests of twelve questions (reading and listening comprehension) with different levels of difficulty were developed. As a result, $60 \%$ of the students exposed to the practices reached a score of 280 to 500 points on the TOEIC standardized test.
\end{abstract}

Key words: CALL, practice, TOEIC, habit, frequency, comprehension. 


\section{Introducción}

Según Ellis (2012), el aprendizaje de un lenguaje se da a través del input (que es toda aquella información que puede ser percibida por los sentidos), el cual es percibido por las personas que desean aprender. Este input tiene dos formas: aural y escrito.

Por otra parte, Ellis (1985) manifiesta que para que un lenguaje sea aprendido, los estudiantes deben tener -además del input- una serie de mecanismos para procesarlo. Esto significa que los estudiantes deben estar expuestos a los diferentes ítems de input del lenguaje para que este se pueda aprender con eficiencia; además, se les debe proveer de las herramientas necesarias para su procesamiento. Existirían dificultades en el aprendizaje si los dos aspectos mencionados no fuesen tomados en cuenta para el aprendizaje.

Esto ayuda a entender por qué los estudiantes de inglés Intermedio I tienen dificultades al momento de aprender inglés, ya que ellos no están expuestos constantemente al input aural y escrito, careciendo además de las herramientas necesarias para el procesamiento del lenguaje. Dando como resultado notas bajas en sus promedios de materia y en la utilización del leguaje de forma eficiente.

La exposición constante al idioma inglés genera mejores resultados en su aprendizaje. Es decir, que el rendimiento de los alumnos podría mejorar si estos fuesen expuestos al lenguaje por mayor tiempo. Van Geert (2012) explica que, la interacción mutua entre dos variables implica que estas se influencian y codeterminan a través del tiempo. De esta manera la exposición de los alumnos al input aural y escrito podría influir en su rendimiento y fluidez en el lenguaje.

El aprendizaje, memoria y la percepción son afectados por la frecuencia de uso. Entre más veces se experimenta algo, se tendrá más presente en la memoria y se recordará con fluidez (Ellis, 2012). Para que los estudiantes incrementen su rendimiento en el aprendizaje del idioma inglés se debe, entonces, ayudar a implementar rutinas que incrementen la frecuencia de la exposición y el uso del lenguaje, sabiendo que ellos carecen de las herramientas y los mecanismos propios para exponerse satisfactoriamente al input del lenguaje.

La exposición al input se dio través del uso de computadoras y la plataforma Moodle. Para poder utilizar este recurso de manera eficiente se consideró la metodología de enseñanza del lenguaje asistido por computadoras $\mathrm{CALL}^{1}$ (conocido por el acrónimo inglés Computer Assisted Language Learning). En palabras de Levy (1997), CALL es la búsqueda y el estudio de aplicaciones en la computadora en la enseñanza y aprendizaje del lenguaje.

1. La autora también hará referencia al término dentro del artículo a través de su acrónimo. 
Una razón para favorecer el enfoque CALL de manera integradora es el papel esencial que asumen los estudiantes. Con relación a este papel, según Hoven (1999) se debe colocar al estudiante en control de su propio aprendizaje en cuanto a contenido, modo, orden, ritmo, nivel y auto-dirección del conocimiento. Se diseñan entonces syllabus enmarcados en una perspectiva teórica sociocultural, en donde los textos son negociables, mediados y hechos para ser interactivos.

Para el aprendizaje de un lenguaje debe de existir una adecuación para el estudiante, la que se define como el número de oportunidades para la implicación con el lenguaje bajo circunstancias apropiadas, basadas en las características del estudiante (Chapelle, 2001). CALL puede ser sensitivo al ritmo del estudiante, el patrón de respuestas y muchos más elementos; los cuales pueden ajustarse al material lingüístico de las necesidades de un individuo (Ahmad et al., 1985).

Además de los aspectos mencionados, en CALL la motivación es vista como un factor esencial en el éxito del aprendizaje del lenguaje. Por lo que Soo (1999) afirma que los usuarios de CALL se han inclinado a señalar que los escenarios en donde hay un uso de la computadora, los ha motivado de gran manera. Igualmente, Pennington (1996) ratifica que los estudiantes ganan motivación a través del uso de la computadora, porque se sienten menos amenazados y son espontáneos, ya que toman más riesgos para aprender.
Por otra parte, una forma de verificar si el progreso de los estudiantes ha sido satisfactorio después de utilizar una metodología de práctica por computadora, es a través de exámenes estandarizados que indican un parámetro del progreso de los estudiantes en el idioma inglés. El Test de Inglés para la Comunicación Internacional o conocido como TOEIC (por sus siglas en inglés Test of English for International Communication) es un recurso valioso que examina las áreas auditiva y lectora; las cuales son precisamente las habilidades que se intentaban desarrollar en los estudiantes de Inglés Intermedio I, utilizando CALL.

El uso de prácticas de computadora ayuda de gran manera para que los estudiantes realicen una tarea, sean menos inhibidos y puedan demostrar sus habilidades en el área de inglés. Retomando todas las ideas planteadas surge la pregunta de investigación: ¿Qué grado de competencia en el idioma inglés y hábitos de estudio se desarrollan en los estudiantes de Inglés Intermedio I al implementar prácticas semanales aurales y de lectura con un enfoque CALL?

\section{Metodología}

El tipo de investigación aplicada fue investigación acción. El problema consistió en que los estudiantes no practicaban el inglés de manera suficiente y apropiada por lo que tampoco desarrollaron habilidades en función del lenguaje. El camino para resolver el problema fue proveer a los estudiantes de prácticas semanales en la forma de asistencia computacional del 
aprendizaje del inglés, proporcionando lecturas, audios y exámenes semanales durante todo el semestre de duración de la materia Inglés Intermedio I. Este tuvo una duración de 19 semanas. Se pretendía que, con la práctica continua, sus hábitos de estudio se incrementaran y la automatización hacia la realización de las prácticas y la exposición mejoraran.

La población total fue de 81 estudiantes, quienes cursaban la materia de Inglés Intermedio I $\mathrm{y}$ estuvieron divididos en cuatro secciones: A, B, C, y D. Para la muestra se seleccionaron las secciones A y C (grupo de intervención), cuyos estudiantes estarían realizando las prácticas de computadora. La sección A tuvo 21 estudiantes y la sección C, 24; en total se tuvo la participación de 45 estudiantes y se determinó como grupo CALL. Las secciones B y D de Inglés Intermedio I formaron parte del grupo control, siendo 36 estudiantes los cuales no estuvieron expuestos al tratamiento CALL.

Se utilizaron textos y audios, tomados de la serie de libros Interchange 1, cuarta edición. Los textos fueron escogidos para que coincidieran con las unidades y contenidos que los estudiantes estaban tomando en sus clases. Los textos eran de un rango de extensión de 300 a 500 palabras; mientras que los audios tuvieron una duración de aproximadamente dos minutos. Se utilizó un total de doce textos y doce audios. El medio para proveer a los estudiantes con este material fue la plataforma Moodle.

\section{Plan de acción de la investigación}

\section{a. Preparación de estudiantes para pruebas de comprensión semanales}

Los estudiantes recibían cada lunes un texto y un audio. Los textos debían leerse cuantas veces fuese necesario, extrayendo el vocabulario desconocido, con el fin de estar preparados para tomar los exámenes. Además, se les pidió escribir doce preguntas relacionadas al audio y al texto en sus cuadernos, para reforzar la preparación respecto a la comprensión lectora y auditiva. Los documentos de los estudiantes eran revisados los días miércoles.

\section{b. Realización de exámenes de comprensión lectora y auditiva}

Los estudiantes tenían que responder a un examen de doce preguntas, durante un tiempo específico; teniendo solo una oportunidad para realizarlo. Se desarrollaron doce exámenes en línea, los cuales tuvieron ocho minutos de duración. Cuando el tiempo finalizaba, el examen se cerraba, guardando las respuestas y proveyendo un puntaje del 1 al 10.

Las preguntas y respuestas fueron diseñadas con distintos niveles de dificultad y diferentes tipos: 1. Vocabulario, donde se tenía que escoger la palabra que hacía falta en la definición o escoger la definición del término; 2. Opción múltiple, una pregunta tres opciones de respuesta; 3. Inferencia, deducción y conclusión basado en evidencia encontrada en el texto, y 4. Preguntas invertidas, en donde se debe elegir la pregunta para la respuesta. 


\section{c. Desarrollo de la prueba TOEIC}

Al final del semestre, 81 estudiantes de Inglés Intermedio I tomaron el examen TOEIC. Los rangos de escala del examen van desde el 0 al 990. Esta prueba se divide en dos secciones:

- La primera es la sección auditiva, que contiene cien preguntas divididas en tres partes: imágenes, conversaciones cortas y discursos largos.

- La segunda es de lectura, que contiene cien preguntas divididas en dos partes: vocabulario y gramática, y textos.

Los estudiantes tomaron el examen durante la semana de parciales, como si este fuera la evaluación final del tercer periodo. De acuerdo al puntaje obtenido se obtuvieron las calificaciones (ver Tabla 1).

Tabla 1. Promedios de aprobación para estudiantes dentro de prueba TOEIC

\begin{tabular}{|c|c|}
\hline $\begin{array}{c}\text { Puntaje obtenido } \\
\text { en TOEIC }\end{array}$ & $\begin{array}{c}\text { Calificación obtenida } \\
\text { según puntaje }\end{array}$ \\
\hline 240 & 6 \\
\hline 250 & 7 \\
\hline 260 & 8 \\
\hline 270 & 9 \\
\hline 280 & 10 \\
\hline
\end{tabular}

\section{Resultados}

El propósito de la investigación fue desarrollar hábitos de estudio en los estudiantes en miras a un mejor desenvolvimiento en el idioma inglés. Después de varias semanas de encuentros frecuentes con textos y audios, así como de exámenes; los estudiantes tomaron el examen estandarizado TOEIC para verificar el nivel de aprendizaje en Inglés Intermedio I.

La tabla 2 presenta la progresión de las calificaciones desde el inicio hasta al fin de la intervención:

Tabla 2. Promedio de calificaciones por periodo

\begin{tabular}{|c|c|c|}
\hline $\begin{array}{c}\text { Primer } \\
\text { período }\end{array}$ & $\begin{array}{c}\text { Segundo } \\
\text { período }\end{array}$ & $\begin{array}{c}\text { Tercer } \\
\text { período }\end{array}$ \\
\hline 6.5 & 7.7 & 8.5 \\
\hline
\end{tabular}

En las semanas que los estudiantes tomaron los exámenes mediante la plataforma Moodle, su desempeño mejoró notablemente. El promedio de las calificaciones de los cuatro exámenes en el Primer período fue de 6.5. Durante la primera fase de aplicación de las prácticas, un 60\% de los estudiantes obtuvieron una calificación por debajo de 6.0 ; un $40 \%$ obtuvo notas de entre 8.0 y 9.0 .

Para el segundo periodo, la mayoría de los estudiantes $(65 \%)$ fue capaz de sacar notas arriba de 6 en los exámenes; por lo cual alcanzaron un promedio de 7.7 ; mientras un $35 \%$ se mantuvo en el promedio de entre 8.0 y 9.0. Durante el tercer periodo hubo un avance notable en el promedio de calificaciones de los exámenes en línea. Esto significó que el 69\% de los estudiantes obtuviera un promedio de 8.5 , y un $31 \%$ de ellos, un puntaje de 8.6 a 9.0.

Al realizar la prueba TOEIC al final de ciclo, los puntajes obtenidos por el grupo CALL fueron diferentes a los del grupo control (ver Figura 1). 


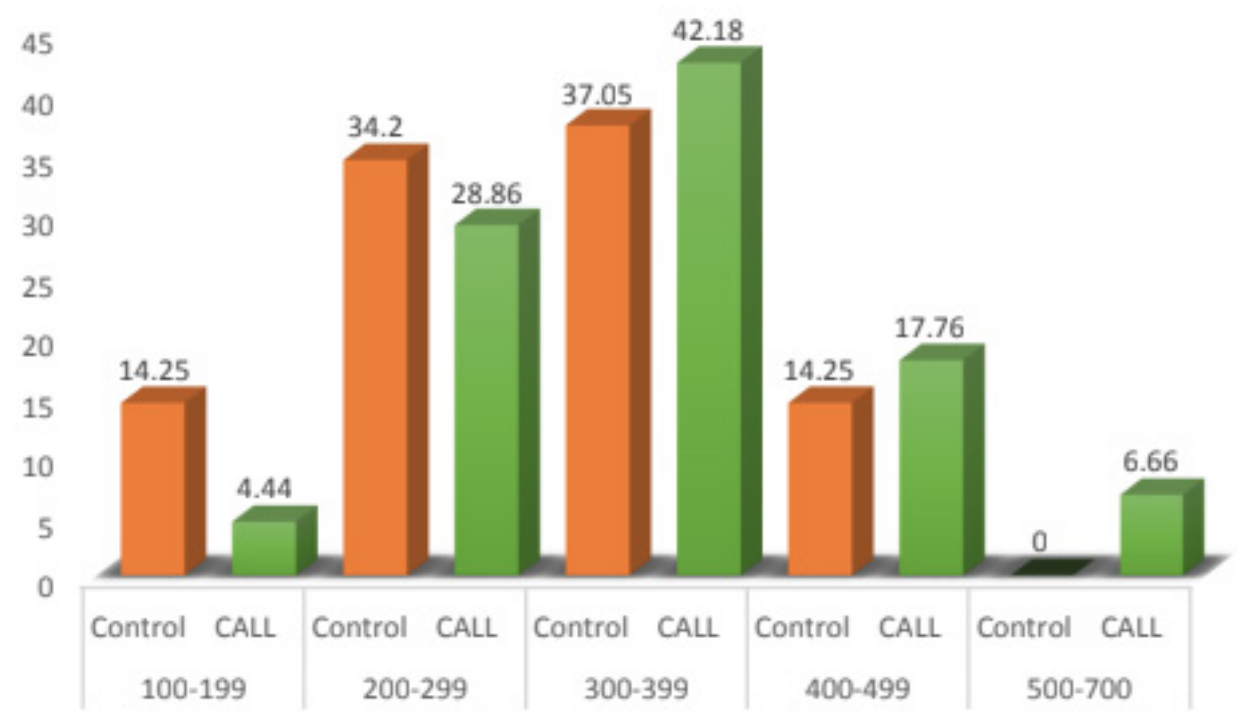

Figura 1. Distribución de puntajes obtenidos por ambos grupos en TOEIC.

Los resultados del grupo perteneciente a la muestra presentaron un puntaje mínimo de 190 y un máximo de 713 puntos, mientras que el grupo control obtuvo un puntaje mínimo de 150 y un máximo de 480. La figura 1 muestra el puntaje promedio obtenido por los dos grupos. Hubo una diferencia considerable entre los estudiantes utilizando el tratamiento CALL, en comparación con aquellos que no estuvieron expuestos al programa. La diferencia radicó en 47 puntos.

Del 100\% de los estudiantes de la muestra, el $71 \%$ obtuvo un puntaje arriba de lo necesario para aprobar la clase (un porcentaje mayor a 290 puntos). De las personas en la muestra que aprobaron con esa cantidad de puntos, 15\% fueron capaces de alcanzar el puntaje máximo para la clase de Inglés Intermedio. A diferencia del grupo control, un $54 \%$ de los estudiantes obtuvo un puntaje de 290 puntos; en donde solo el 5\% fue capaz de conseguir el puntaje requerido para Inglés Intermedio I.
Por otra parte, hubo un porcentaje de estudiantes de ambos grupos que no obtuvo la cifra necesaria para aprobar la clase. En el grupo control, el 17\% de los estudiantes no aprobó, ya que los puntajes se situaron por debajo los de 90 puntos. En el caso del grupo muestra, el $10 \%$ no alcanzó la cifra requerida, teniendo calificaciones por debajo de los 50 puntos.

Como se aprecia en la figura 1, la mayoría de los puntajes están entre 200 a 399 puntos, con muy pocas personas en ambos extremos. Un dato interesante fue que el grupo control obtuvo un porcentaje mayor en el más bajo percentil y ningún puntaje alto; caso contrario a la muestra, en donde se obtuvieron muchas puntuaciones en el percentil más alto. En ambos grupos, las puntuaciones obtenidas presentaron una tendencia positiva en cuanto el aprendizaje de los estudiantes. 
El uso de prácticas por computadora semanales se contrastó con la realización del examen TOEIC, (figura 2), mostrando los puntajes del grupo que estuvo bajo el tratamiento CALL y el grupo control.

En la figura 2 se destacan el rendimiento de los estudiantes expuestos a las prácticas por computadora y el de los estudiantes que no realizaron las prácticas semanales. Los puntajes obtenidos superaron a los esperados que eran de 280 para Inglés Intermedio I. Para el caso, las puntuaciones más altas fueron obtenidas por el grupo que estuvo bajo las prácticas de computadora, logrando puntajes arriba de 280 .

\section{Discusión}

El promedio estándar para la muestra de Inglés Intermedio I fue de 335 puntos en el examen TOEIC, lo cual es 45 puntos más que el rango de puntaje esperado para esa clase. Esto significa que el nivel de preparación de los estudiantes con el programa les brindó ventaja al momento de aplicar la prueba de estandarizada TOEIC.
Es importante mencionar que el grupo control también mostró mejoría, pero en comparación con el grupo CALL, estos estudiantes tuvieron bajas puntuaciones en general.

Para lograr la estimulación necesaria para aprender, según Soo (1999), "se puede utilizar CALL, el cual motiva de gran manera a los estudiantes en cuanto a la intención y el aprendizaje" (p.12). Los resultados de la muestra y el grupo control presentan el progreso de las habilidades aural y lectora. La implicación es clara ya que, al utilizar el programa de práctica por computadora, los estudiantes alcanzaron un promedio de 47 puntos extras en los resultados de su examen TOEIC. Esta evidencia puede motivar a los estudiantes para trabajar activamente en un programa similar al que acá se documenta.

Según Saegusa (1985), "por cada punto que se alcanza en el TOEIC, esto refiere a trabajar una hora aprendiendo inglés" (p. 67). Esto significa que los estudiantes obtuvieron el equivalen-

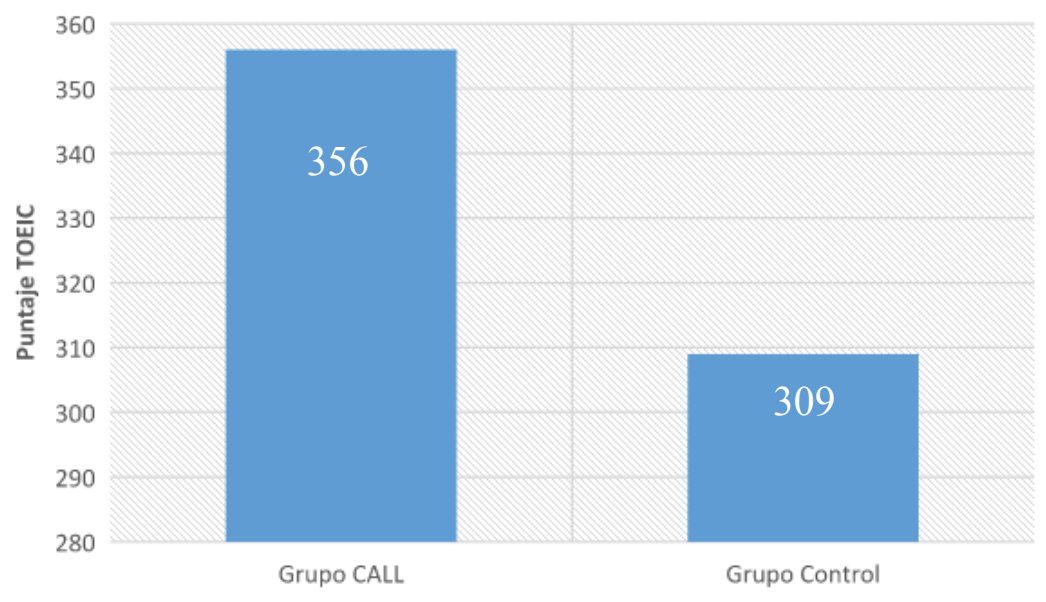

Figura 2. Promedio de notas finales TOEIC. 
te a estudiar 47 horas extras al llevar a cabo el programa indicado. Si la materia de Inglés Intermedio I posee un total de 80 horas de trabajo, el dato registrado durante la aplicación de la estrategia implicaría que los estudiantes asimilaron -por ellos mismos- el equivalente a la mitad de todas las horas clase que se deben estudiar en la asignatura.

Una característica de la formación de hábitos es que, a medida que estos se practican con regularidad, resultan ser más fáciles en el tiempo (Wood et al, 2002). Durante el estudio, al a los estudiantes les fue difícil, inicialmente, realizar las pruebas, tomarse el tiempo para estudiar y desarrollar las actividades asignadas; pero conforme fueron avanzando, el esfuerzo disminuyó. Los estudiantes automatizaron el desarrollo de las actividades, y con el tiempo, la dificultad fue mínima, ya que se estableció una rutina.
Esto se puede evidenciar no solamente por los resultados del examen TOEIC, sino también porque el promedio de los estudiantes en el primer período de aplicación fue de 6.5; durante el segundo fue de $7.7 \mathrm{y}$ el tercero, de 8.5. Esto demuestra habilidad y facilidad por parte de los estudiantes para realizar los exámenes; en consecuencia, una automatización de las prácticas.

En conclusión, es oportuno asegurar que, si se provee de un programa de prácticas bien establecido con diversidad de material y evaluaciones frecuentes, además de permitir a los estudiantes asimilar el contenido y desarrollar habilidades competentes en inglés, durante el nivel Intermedio I, se obtendrían mejores promedios en exámenes estandarizados como TOEIC; que con una metodología que no tenga dicha estructura.

\section{Referencias}

Aarts, H. y Dijksterhuis, A. (2000). Habits as knowledge structures: Automaticity in goal-directed behavior. Journal of Personality and Social Psychology, 78, 53-63.

Ahmad, K.; Corbett, G.; Rogers, M. y Sussex, R. (1985). Computers, Language Learning and Language Teaching. Cambridge: Cambridge University Press.

Bygate, M.; Skehan, P. y Swain, M. (2001). Introduction. In M. Bygate, P. Skehan y M. Swain (eds.), Researching pedagogic tasks: second llanguage learning, teaching and testing (120). Harlow, UK: Pearson.

Chapelle, C. A. (2001). Computer applications, in second language acquisition. Cambridge: Cambridge University Press.

Corbel, C. (1999). Task as tamogotchi: ESL teachers 'work in the emerging hypermedia environment. Prospect, 14, 40-5. 
Danner, U.N.; Aarts, H. y de Vries, N. K. (2008). Habit vs. intention in the prediction of future behaviour: The role of frequency, context stability and mental accessibility of past behaviour. British Journal of Social Psychology, 47, 245-265.

Dörnyei, Z. (2001). Motivational strategies in the language classroom. Cambridge: Cambridge University Press.

Duhigg, C. (2014). The Power of Habit Why we do what we do in Life and Bussines. New York: Radom House.

Ellis, N. C. (2002). Frequency Effects in Language Processing: A Review with Implications for Theories of Implicit and Explicit Language Acquisition. Studies in Second Language Acquisition, 24(2), 143-188.

Hoven, D. (1999). CALL-ing the learner into focus: towards a learner centered model. In R. Debski y M. Levy (eds.), World CALL: global perspectives on computer assisted language learning (149-67): Lisse: Swets \& Zeitlinger.

Levy, M. (1997). Computer-assisted languagelearning: context and contextualisation. Oxford: Oxford University Press.

O’Doherty, J. y Balleine, B. (2010). Human and rodent homologies in action control: corticostriatal determinants of goal-directed and habitual action. Neuropsychopharmacology, 35, 48-69.

Pennington, M. C. (1996) The power of the computer in language education. In M. C. Pennington (ed.) The power of CALL (pp. 1-14). Houston: Athelstan.

Runger, D. y Wood, W. (2015). Maintenance of healthy behaviors: forming and changing habits. In Behavioral Economics and Public Health (ed.) C. Roberto, I. Kawachi. Oxford, UK: University of Oxford.

Saegusa, Y. (1985). Prediction of English Proficiency Progress. Musashino English and American Literature, 18. Tokyo: Musashino Women's University.

Soo, K. (1999). Theory and research: learning styles, motivaton and the CALL classroom. In J.

Van Geert, P. (1994). Dynamic Systems of Development: Change Between Complexity and Chaos. New York; Harvester Wheasheaf.

Wood, W. y Neal, D. (2009). The habitual consumer. Journal of Consumer Psychology, 19(4), 579-592.

Wood, W.; Quinn, J. M. y Kashy, D. A. (2002). Habits in everyday life: thought, emotion, and action. J. Personal. Soc. Psychol. 